\title{
Analysis of the etiologies of female infertility in Yunnan minority areas
}

\author{
Fen Zhang ${ }^{1,2+}$, Qing Feng ${ }^{3 \dagger}$, Linna Yang ${ }^{1,2}$, Xuelian Liu ${ }^{5}$, Lingyun Su ${ }^{1,2}$, Chunyan Wang $^{6}$, Huimei Yao , \\ Dawei Sun ${ }^{4^{*}}$ and Yun Feng ${ }^{1,2^{*}}$
}

\begin{abstract}
Background: The present study aims to provide a comparative analysis of the etiologies of female infertility between Dehong, on the Yunnan Frontier, and Kunming.

Methods: A retrospective study, which included 941 infertile females in Kunming who were treated in the First People's Hospital of Yunnan Province and infertile females who were treated in the local hospital in Dehong from January 2016 to November 2018, was conducted. A comparative analysis of the etiologies of infertility in the two regions was then carried out.

Results: In patients with primary infertility, ovulation disorder (15.03\%) was the main cause of infertility in Kunming, and pelvic inflammatory disease (25.59\%) was the main cause in Dehong. With regard to secondary infertility, although pelvic inflammatory disease was the main cause of infertility in both regions, the incidence of intrauterine adhesions in Kunming was significantly higher than in Dehong.

Conclusions: The etiology of infertility showed different epidemiological characteristics depending on the region, hence individualized treatment should be given accordingly
\end{abstract}

Keywords: Infertility, Etiology, Related factors, Yunnan, Minority areas

\section{Background}

According to criteria issued by the World Health Organization (WHO), those who are married, have a normal sex life without the use of contraception, and have lived with their partner for one year without conception are called infertile [1]. Due to influence from a given region's environment, climate, culture, and customs, the incidence of infertility differs across regions and countries. It is reported that the incidence of infertility in developed

\footnotetext{
*Correspondence: dawei_sun66@126.com; fengyuncnfy@163.com

${ }^{\dagger}$ Fen Zhang and Qing Feng contributed equally to this study

${ }^{1}$ Department of Obstetrics and Gynecology, The First People's Hospital of Yunnan Province, No. 157 of Jinbi Road, Xishan District, Kunming 650032, China

${ }^{4}$ Department of Obstetrics and Gynecology, Peking Union Medical College Hospital (Dongdan Campus), No.1 Shuaifuyuan Wangfujing, Dongcheng District, Beijing 100730, China

Full list of author information is available at the end of the article
}

countries is $5-8 \%$, while in developing countries it is as high as $5.8-44.2 \%$. This difference is also reflected in various specific countries. For example, the incidences of infertility in Iran, Britain, and China range between $3-8 \%, 2-26 \%$, and $1-18 \%$, respectively [2].

Infertility is regarded as a global problem that affects the health and economic situations of sufferers and their families $[3,4]$. The inability to have a child whenever they want, can lead to a crisis situation in women because there are personal, interpersonal, social and religious expectations of having children. In developing countries, infertility causes social stigma, women diagnosed with infertility are abandoned by their husbands, become victims of spousal and family violence, are excluded from the family and society and experience a sense of failure [5].

Previous studies have revealed that differences in the etiologies of infertility are mainly due to differences in

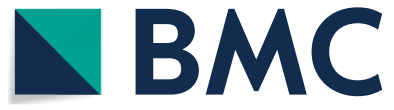

(c) The Author(s) 2021. This article is licensed under a Creative Commons Attribution 4.0 International License, which permits use, sharing, adaptation, distribution and reproduction in any medium or format, as long as you give appropriate credit to the original author(s) and the source, provide a link to the Creative Commons licence, and indicate if changes were made. The images or other third party material in this article are included in the article's Creative Commons licence, unless indicated otherwise in a credit line to the material. If material is not included in the article's Creative Commons licence and your intended use is not permitted by statutory regulation or exceeds the permitted use, you will need to obtain permission directly from the copyright holder. To view a copy of this licence, visit http://creativecommons.org/licenses/by/4.0/. The Creative Commons Public Domain Dedication waiver (http://creativecommons.org/publicdomain/zero/1.0/) applies to the data made available in this article, unless otherwise stated in a credit line to the data. 
culture, socioeconomics, health care practices, policies, and environmental conditions [6]. The present study provided a comparative analysis of the etiologies of female infertility between Dehong, on the Yunnan Frontier, and Kunming. This study aimed to determine whether infertile Han females from different regions had different epidemiological characteristics.

\section{Methods}

\section{Study population and sample size}

This is a cross-sectional study. The random sample selection was used. From January 2016 to November 2018, 941 infertile Han females in Kunming who were treated in the First People's Hospital of Yunnan Province, and 813 infertile females who were treated in Dehong Dai and Jingpo Autonomous Prefecture Hospital, were enrolled in the study.

\section{Inclusion criteria}

All the patients met the WHO criteria for the diagnosis of female infertility: having been sexually active for at least 12 months without using contraception without becoming pregnant. Among them, those who have never been pregnant previously fall under the category of primary infertility; those who have been pregnant in the past but who have not been pregnant for 12 consecutive months without contraception fall under the category of secondary infertility.

All patients had normal cognitive function and participated in the study voluntarily with signed informed consent.

\section{Exclusion criteria}

Exclusion criteria for this study were infertility due to the male component, less than one year of menarche or entrance into the perimenopausal period as verified by the detection of sex hormones, malignant tumors, severe cardiovascular, cerebrovascular, hepatic, or renal disease, mental disease or cognitive impairment, unwillingness to sign informed consent, and relocation from other places to the survey sites.

\section{Data collection}

Medical records were obtained from doctors in the two hospitals. A proforma used to extract information from the patient records. The contents of clinical data included age, educational background, nationality, residence, body mass index (BMI), marriage age, duration of disease, menstrual history, previous history of infertility, and reproductive history. The actual etiologies of infertility were obtained from the operations themselves, and the etiologies and related factors of infertility in the two regions were compared and analyzed.

\section{Statistic analysis}

An excel spreadsheet was formulated and the collected medical history data were input by recruiting and reexamination. For those cases that required surgery, the intraoperative conditions were recorded. Double entry was adopted for data input. In case of any discrepancy in data entry, the original inquiry questionnaire and medical record data were rechecked.

SPSS 22.0 software was used for data analysis, the Pearson $\chi^{2}$ test was used for comparing proportions, and logistic regression analysis was adopted for the analysis of the influencing factors of infertility. P value $<0.05$ was regarded as statistically significant.

\section{Results}

\section{General characteristics}

From the 1,754 patients analyzed in the present study, 941 were in Kunming and 813 in Dehong. There was no statistical significance in the age distribution of infertile patients between the two regions $\left(x^{2}=7.42\right.$, $P$ value $=0.192$ ). The mean age of infertile patients in Kunming was $29.09 \pm 4.81$ years (range 20-41 years), and the mean age of infertile patients in Delong was $28.87 \pm 5.02$ years (range 19-44 years); the difference of age between the two regions was not statistically significant $(P$ value $=0.349$ ). The difference in the distribution of educational background, however, was statistically significant $\left(\chi^{2}=31.87, P\right.$ value $\left.<0.001\right)$; there were more infertile patients with a high educational background (college degree or above) in Kunming than in Dehong. Statistical significance was also found in the distribution of residence in the two regions $\left(x^{2}=9.71\right.$, $P$ value $=0.021$ ); the majority of the infertile patients (75.24\%) in Kunming lived in urban areas, whereas the majority in Dehong (77.49\%) lived in rural areas. Statistical analysis showed that there was a significant difference in the distribution of marriage age between the two regions $\left(\chi^{2}=23.19, \mathrm{P}\right.$ value $\left.<0.001\right)$; the percentage of patients with a marriage age of $\leq 25$ years in the Dehong region $(66.18 \%)$ was significantly higher than in Kunming (48.54\%). There was no significant difference in the number of sexual encounters per week between the two regions $(3.23 \pm 1.81$ vs. $3.12 \pm 2.10, \mathrm{P}$ value $=0.287)$. In addition, among the patients in Kunming, the number of patients who knew their most fertile days of the month was higher than in the Dehong area; the difference was statistically significant $\left(\chi^{2}=31.52\right.$, $P$ value $\left.<0.001\right)$.

The majority of infertile patients in both regions had a BMI within the normal range, but the BMI of patients in Kunming was higher than in Dehong. There were more lean patients $(\mathrm{BMI}<18.5)$ in Dehong than that in Kunming, and there were more overweight patients 
Table 1 Comparison of BMI in patients with infertility between the two regions $(n=1754)$

\begin{tabular}{|c|c|c|c|c|}
\hline \multirow[t]{2}{*}{$\mathrm{BMI}\left(\mathrm{kg} / \mathrm{m}^{2}\right)$} & \multicolumn{2}{|c|}{$\begin{array}{l}\text { Kunming region } \\
(n=941)\end{array}$} & \multicolumn{2}{|c|}{$\begin{array}{l}\text { Dehong region } \\
(n=813)\end{array}$} \\
\hline & Case (n) & Percent (\%) & Case (n) & Percent (\%) \\
\hline Underweight $(<18.5)$ & 98 & 10.47 & 174 & 21.49 \\
\hline Normal (18.5-24.9) & 664 & 70.51 & 523 & 64.33 \\
\hline Overweight ( $\geq 25$ ) & 179 & 19.02 & 116 & 14.27 \\
\hline
\end{tabular}

$B M /$ body mass index

Table 2 The distribution of past history in infertile patients between the two regions $(n=1754)$

\begin{tabular}{|c|c|c|c|c|}
\hline \multirow[t]{2}{*}{ Medical histories } & \multicolumn{2}{|c|}{$\begin{array}{l}\text { Kunming region } \\
(n=941)\end{array}$} & \multicolumn{2}{|c|}{$\begin{array}{l}\text { Dehong region } \\
(n=813)\end{array}$} \\
\hline & Case (n) & Percent (\%) & Case (n) & Percent (\%) \\
\hline Pelvic inflammatory & 56 & 5.95 & 127 & 15.62 \\
\hline Ectopic pregnancy & 68 & 7.22 & 57 & 7.01 \\
\hline Ovarian cysts & 49 & 5.21 & 40 & 4.92 \\
\hline PCOS & 83 & 8.82 & 43 & 5.29 \\
\hline Adenomyosis & 11 & 1.17 & 16 & 1.97 \\
\hline Endometriosis & 20 & 2.13 & 39 & 4.80 \\
\hline Endometrial polyp & 19 & 2.02 & 13 & 1.6 \\
\hline Intrauterine adhesions & 29 & 3.08 & 15 & 1.85 \\
\hline Cervicitis & 81 & 8.61 & 82 & 10.86 \\
\hline Vaginitis & 24 & 2.55 & 51 & 6.27 \\
\hline
\end{tabular}

PCOS polycystic ovary syndrome

(BMI $\geq 25)$ in Kunming than that in Dehong (see Table 1). The difference in BMI between the two regions was statistically significant $\left(\chi^{2}=43.32\right.$, P value $\left.<0.001\right)$.

\section{Comparison of medical histories}

Table 2 shows that, in the medical histories of the patients in Kunming, polycystic ovarian syndrome (PCOS) accounted for the highest proportion $(8.82 \%)$, followed by cervicitis ( $8.61 \%)$, ectopic pregnancy $(7.22 \%)$, and pelvic inflammatory disease (5.95\%). Medical histories for patients in Dehong showed that the proportion of pelvic inflammatory disease (15.62\%) was the highest, followed by cervicitis (10.86\%), ectopic pregnancy $(7.01 \%)$, and vaginitis $(6.27 \%)$.

\section{Analysis of pregnancy histories for patients with secondary infertility}

As shown in Table 3, the pregnancy times for patients with secondary infertility differed between regions, and this difference was statistically significant. In Kunming, the number of patients with a history of more than two previous pregnancies was significantly higher than in Dehong. In Dehong, however, the majority of patients with secondary infertility had a history of only one previous pregnancy.

\section{Analysis of factors influencing infertility in patients}

Logistic regression models of the factors influencing infertility were established in both locations. Based on the data from infertile patients in Dehong, the regression equation was used for the comparison between the Kunming and non-Kunming (Dehong) areas. Then, based on the data of infertile patients in Kunming, the regression equation was used for the comparison between Dehong and non-Dehong (Kunming) areas. The results were showed in Table 4. In the regression equation, marriage age, BMI, menstrual flow are the quantitative data; Menstrual regularity and pelvic inflammatory are the categorical data.

In Kunming region, patients with older marriage age (odds ratio 2.716, P value $=0.032$ ), higher BMI (odds ratio 3.293 , $\mathrm{P}$ value $=0.024$ ), irregular menstruation (odds ratio $1.924, \mathrm{P}$ value $=0.016$ ), and less menstrual volume (odds ratio $2.392, \mathrm{P}$ value $=0.001$ ) were more likely to be infertile compared to those without. In Dehong region, patients who had pelvic inflammatory disease were almost four times likely to be infertile compared to those without (odds ratio 3.716, P value $<0.001$ )

Table 3 The constituent ratio of pregnancy history in patients with secondary infertility between the two regions $(n=1089)$

\begin{tabular}{|c|c|c|c|c|c|c|}
\hline \multirow[t]{2}{*}{ Groups } & \multicolumn{2}{|c|}{ Kunming region $(n=655)$} & \multicolumn{2}{|c|}{ Dehong region $(n=434)$} & \multirow[t]{2}{*}{$x^{2}$} & \multirow[t]{2}{*}{$P$ value } \\
\hline & Case (n) & Percent (\%) & Case (n) & Percent (\%) & & \\
\hline G1 & 244 & 37.25 & 252 & 58.03 & 32.79 & $<0.001$ \\
\hline G2 & 202 & 30.84 & 106 & 24.45 & & \\
\hline G3 & 112 & 17.10 & 42 & 9.49 & & \\
\hline$\geq \mathrm{G} 4$ & 97 & 14.81 & 34 & 8.03 & & \\
\hline
\end{tabular}

G, generation; $\chi^{2}$, Chi-square 
Table 4 Logistic analysis of multiple factors between the two regions $(n=1754)$

\begin{tabular}{llllllll}
\hline Index & B & S.E & Wald & df & sig & OR & 95\% Cl of OR \\
\hline Kunming region $(n=941)$ & & & & & & & \\
Age of marriage & 0.999 & 0.037 & 5.903 & 1 & 0.032 & 2.716 & $2.526-2.920$ \\
BMl & 1.192 & 0.173 & 9.572 & 1 & 0.024 & 3.293 & $2.346-4.623$ \\
Menstrual regularity & 0.654 & 0.319 & 6.041 & 1 & 0.016 & 1.924 & $1.029-3.594$ \\
$\quad$ Menstrual flow & 0.872 & 0.312 & 10.412 & 1 & 0.001 & 2.392 & $1.298-4.408$ \\
Dehong region ( $\mathrm{n}=813)$ & & & & & & & \\
$\quad$ Pelvic inflammatory & 1.313 & 0.157 & 17.305 & 1 & $<.001$ & & $2.733-5.057$ \\
\hline
\end{tabular}

Cl confidence interval, $O R$ odds ratio

\section{Etiologies of infertility the two regions}

Among the 1,754 infertile patients included in the present study, 1,257 (71.66\%) had undergone laparoscopy combined with hysteroscopy. Among them, pathologies were found in 1,234 patients, accounting for $98.17 \%$ of the total number of infertile patients; 432 cases (33.41\%) had pelvic inflammatory disease, 311 cases (24.74\%) had hydrosalpinx, 251 cases (19.97\%) had an ovarian cyst, 237 cases (18.85\%) had hysteromyoma, 165 cases $(13.13 \%)$ had intrauterine adhesions, and 147 cases $(11.69 \%)$ had PCOS. Of these 1,257 cases of infertility, $366(20.05 \%)$ possessed two or more of the factors that contribute to infertility. Details are shown in Table 5.
Etiologies of infertility in patients with primary infertility in the two regions

Among the etiologies of primary infertility in the two regions, ovulation disorder (36.71\%) was the main cause in Kunming, while pelvic inflammatory disease (25.59\%) was the main cause in Dehong. Additionally, the proportions of tubal factor (P value $=0.034)$ and pelvic tuberculosis $(P$ value $=0.007)$ in Dehong were much higher than in Kunming, as can be seen in Table 6.

\section{Etiologies of infertility in patients with secondary infertility} in the two regions

As shown in Table 7, among the etiologies of secondary infertility, the proportion of pelvic inflammatory disease $(28.88 \%$ vs. $23.66 \%, \mathrm{P}$ value $=0.021)$ and tubal

Table 5 Comparison of the etiologies of infertility through exploratory operation by laparoscopy combined with hysteroscopy between the two regions

\begin{tabular}{|c|c|c|c|c|}
\hline Pathology & Total $(n=1257)$ & Kunming $(n=651)$ & Dehong $(n=606)$ & $P$ value \\
\hline Pelvic inflammatory & $432(33.41 \%)$ & $198(30.34 \%)$ & $222(36.63 \%)$ & 0.037 \\
\hline Slightly adhesion & $165(13.13 \%)$ & $79(12.14 \%)$ & $86(14.19 \%)$ & 0.082 \\
\hline Moderate adhesion & $155(12.09 \%)$ & $73(11.21 \%)$ & $79(13.04 \%)$ & 0.297 \\
\hline Severe adhesion & $103(8.19 \%)$ & $46(7.07 \%)$ & $57(9.41 \%)$ & 0.021 \\
\hline Hydrosalpinx obstruction & $311(24.74 \%)$ & $141(21.66 \%)$ & $170(28.07 \%)$ & 0.021 \\
\hline Unilateral obstruction & $174(13.84 \%)$ & $84(12.96 \%)$ & $90(14.85 \%)$ & 0.216 \\
\hline Bilateral obstruction & $137(10.90 \%)$ & $57(8.76 \%)$ & $80(13.20 \%)$ & 0.042 \\
\hline Endometriosis & $140(11.14 \%)$ & 71 (10.91\%) & $69(11.39 \%)$ & 0.932 \\
\hline Simple cyst of ovary & $93(7.40 \%)$ & $51(7.83 \%)$ & $42(6.93 \%)$ & 0.312 \\
\hline Chocolate cyst of ovary & $151(12.01 \%)$ & 73 (11.21\%) & $78(12.87 \%)$ & 0.571 \\
\hline Myoma of uterus & $237(18.85 \%)$ & $122(18.74 \%)$ & 115 (18.98\%) & 0.365 \\
\hline PCOS & $147(11.69 \%)$ & $84(12.90 \%)$ & $63(10.40 \%)$ & 0.897 \\
\hline Intrauterine adhesions & $165(13.13 \%)$ & $111(17.05 \%)$ & $54(8.91 \%)$ & 0.019 \\
\hline Endometrial polyp & $113(8.99 \%)$ & $59(9.06 \%)$ & $54(8.91 \%)$ & 0.953 \\
\hline Pelvic tuberculosis & $51(4.06 \%)$ & $9(1.38 \%)$ & $42(6.93 \%)$ & 0.002 \\
\hline Uterine malformation & $20(1.59 \%)$ & $11(1.69 \%)$ & $9(1.49 \%)$ & 0.629 \\
\hline Teratoma of ovary & 7 (0.56\%) & 4 (0.614\%) & $3(0.50 \%)$ & 0.891 \\
\hline No complications & $23(1.83 \%)$ & $14(2.15 \%)$ & $9(1.49 \%)$ & 0.738 \\
\hline
\end{tabular}

PCOS polycystic ovary syndrome 
Table 6 Comparison of the etiologies of primary infertility between the two regions

\begin{tabular}{lcll}
\hline Pathogeny & Kunming (n=286) & Dehong (n=379) & P value \\
\hline Pelvic inflammatory & $36(12.59 \%)$ & $97(25.59 \%)$ & 0.013 \\
Tubal factor & $43(15.03 \%)$ & $76(20.05 \%)$ & 0.034 \\
Ovulation disorders & $105(36.71 \%)$ & $67(17.68 \%)$ & 0.001 \\
Endometriosis & $13(4.55 \%)$ & $29(7.65 \%)$ & 0.892 \\
Ovarian cysts & $28(9.79 \%)$ & $50(13.19 \%)$ & 0.372 \\
Myoma of uterus & $27(9.44 \%)$ & $47(12.40 \%)$ & 0.127 \\
Intrauterine adhe- & $10(3.49 \%)$ & $19(5.01 \%)$ & 0.772 \\
$\quad$ sions & & & \\
Endometrial polyp & $13(4.57 \%)$ & $23(6.07 \%)$ & 0.561 \\
Pelvic tuberculosis & $9(3.15 \%)$ & $42(11.08 \%)$ & 0.007 \\
Uterine malforma- & $4(1.42 \%)$ & $4(1.05 \%)$ & 0.814 \\
$\quad$ tion & & & \\
Immune factors & $35(12.24 \%)$ & $40(10.55 \%)$ & 0.953 \\
Unknown cause & $9(3.15 \%)$ & $6(1.61 \%)$ & 0.219 \\
\hline
\end{tabular}

Table 7 Comparison of the etiologies of secondary infertility between the two regions

\begin{tabular}{lccc}
\hline Pathogeny & Kunming $(\mathbf{n = 6 5 5 )}$ & Dehong $(\mathbf{n = 4 3 4 )}$ & P value \\
\hline Pelvic inflammatory & $155(23.66 \%)$ & $125(28.88 \%)$ & 0.021 \\
Tubal factor & $105(16.04 \%)$ & $94(21.61 \%)$ & 0.036 \\
Ovulation disorders & $80(12.21 \%)$ & $54(12.44 \%)$ & 0.539 \\
Endometriosis & $58(8.85 \%)$ & $40(9.22 \%)$ & 0.872 \\
Ovarian cysts & $64(9.77 \%)$ & $46(10.59 \%)$ & 0.307 \\
Myoma of uterus & $95(14.66 \%)$ & $68(15.67 \%)$ & 0.105 \\
Intrauterine adhe- & $101(15.42 \%)$ & $35(8.06 \%)$ & 0.014 \\
$\quad$ sions & & $31(7.14 \%)$ & 0.873 \\
Endometrial polyp & $46(7.02 \%)$ & $5(1.15 \%)$ & 0.923 \\
Uterine malforma- & $7(1.07 \%)$ & & \\
tion & & $46(10.59 \%)$ & 0.795 \\
Immune factors & $70(10.68 \%)$ & $3(0.69 \%)$ & 0.614 \\
Unknown cause & $5(0.76 \%)$ & & \\
\hline
\end{tabular}

factor $(21.61 \%$ vs. $16.04 \%$, P value $=0.036)$ in Dehong was significantly higher than in Kunming, while the proportion of intrauterine adhesions in Kunming was significantly higher than in Dehong $(15.42 \%$ vs. $8.06 \%$, P value $=0.014)$.

\section{Discussion}

The present study revealed that infertile patients in Kunming were mainly affected by such risk factors as late marriage age, being overweight, irregular menstruation, and less menstrual volume, while the infertile patients in Dehong were mainly influenced by the risk factor of previous pelvic inflammatory disease. The differences in etiologies between the two regions were mainly reflected in four factors, including pelvic inflammatory disease, hydrosalpinx, PCOS, and pelvic tuberculosis.

Pelvic inflammation includes the infection and inflammation of the upper genital tract (endometrium, fallopian tubes, ovaries, and pelvic peritoneum) [7-9], which is liable to occur in sexually active females. Becoming sexually active too early, not attending sufficiently to personal hygiene, and sexual activity during menstruation are all factors that make the pelvic cavity prone to invasion by bacteria from the vagina, resulting in pelvic inflammation. The earlier sexual activity is begun, the higher the proportion of pelvic inflammation [10]. The risk factors for pelvic inflammation are having multiple sexual partners, youth, smoking, and using illicit drugs [11]. In recent years, the incidence of pelvic inflammatory disease in China has increased yearly. The results of this clinical investigation showed that the proportion of infertility in patients with pelvic inflammatory disease was about $20-40 \%$. The proportion and duration of occurrences of pelvic inflammatory disease were closely correlated with the proportion of infertility [12].

Pelvic inflammatory disease causes infertility by changing the topography of the pelvic cavity and the internal structure of the fallopian tubes, and ovulation disorders are caused by inflammation around the ovary [13]. In addition, some experts have pointed out that the amount and some characteristics of vaginal secretions in patients with pelvic inflammation might lead to changes in the acid-base environment, which ultimately affects sperm's activity, thus interfering with fertilization.

In the current study, from the 1,754 infertile patients, 432 had pelvic inflammatory disease, accounting for $24.63 \%$ of the total number of patients. The proportion of infertile patients with previous pelvic inflammatory disease in Dehong (15.62\%) was higher than in Kunming $(5.95 \%)(\mathrm{P}<0.05)$. This difference may be correlated with the medical and health conditions, the poor sexual health consciousness, and the younger marriage age in Dehong. Thus, we should note the levels and prevalence of sexual health and hygiene education for childbearing-age couples in Dehong.

Common diseases leading to ovulation disorders in females of childbearing age are PCOS and hyperprolactinemia. Among such females, the incidence of PCOS ranges from 5 to $10 \%$, and the incidence in infertile patients is as high as $30-60 \%$ [14]. The phenotype of PCOS is affected by obesity, which also intensifies the metabolic characteristics of PCOS [15]. In the present study, the proportion of ovulation disorders was $17.45 \%$, with a proportion of as high as $36.71 \%$ in patients with primary infertility in Kunming. The difference in proportion of ovulation disorders between the two regions may be closely correlated with the differences in BMI, diet 
structure, work pressure, and endocrine disorders caused by an erratic sleep schedule. Therefore, emphasis should be placed on a healthy lifestyle, moderate exercise regulation, and reasonable diet for the females of childbearing age in Kunming in order to reduce the proportion of ovulation disorders.

Tubal infertility, of which hydrosalpinx is a major cause, accounts for $30-50 \%$ of patients with infertility [16]. Inflammation inside the fallopian tube is caused by external infection, salpingitis, or nodular salpingitis (thickening or scarring of the tubal nodules in the isthmus) [17]. Salpingitis may cause an obstruction in and permanent functional injury to the distal end of the fallopian tube. The most common cause of salpingitis is sexually transmitted pelvic inflammation. The percentage of infertility caused by fallopian tube issues in Dehong was much higher than in Kunming, and its main influencing factor was pelvic inflammation. The prevention strategy in this case should thus be similar to that of pelvic inflammation. Actively popularizing reproductive health education and reducing the incidence of sexually transmitted diseases and premarital abortions may have a preventative effect on the reduction of infertility caused by fallopian tube and pelvic diseases.

It has been reported that the incidence of infertility in patients with endometrial adhesions is as high as $90 \%$ [18]. Uterine cavity normalcy and sufficient endometrial thickness are essential for a successful pregnancy $[19,20]$. Intrauterine adhesions are caused by injury of the basement layer of the endometrium caused by postoperative or inflammatory infection of the uterine cavity, such as abortion and curettage. Scar tissue formed in the uterine cavity can partially or completely occlude the normal cavity, resulting in menstrual abnormalities, infertility, recurrent abortion, and other complications. In the present study, the proportion of intrauterine adhesion in patients with secondary infertility in Kunming (15.42\%) was much higher than in Dehong $(8.06 \%)(\mathrm{P}<0.05)$. In addition, the number of previous pregnancies in patients with secondary infertility in the two regions was different, and this difference was statistically significant. There were significantly more patients with more than two previous pregnancies in Kunming than in Dehong, while the majority of patients with secondary infertility in Dehong were those with only one previous pregnancy $(\mathrm{P}<0.05)$. This might be correlated with the high abortion rate found in infertile patients in Kunming [21]. Thus, according to this difference, we can carry out scientific contraception for females in Kunming, reduce the accidental pregnancy, and weaken the injury of operation to the uterine cavity.

This study has several limitations. First, the crosssectional nature of the study design might not show the cause and effect relationships between study variables. Second, the samples for this study were taken from the only one teaching hospital of each area. Finally, the sample size of this study was not calculated based on a certain equation.

\section{Conclusion}

In conclusion, there were marked differences in the etiologies and risk factors of infertility between women living in Kunming and Dehong.

\section{Abbreviations \\ WHO: World Health Organization; BMI: Body mass index; PCOS: Polycystic ovarian syndrome.}

\section{Acknowledgements}

We would like to acknowledge the hard and dedicated work of all the staff that implemented the intervention and evaluation components of the study.

\section{Authors' contributions}

FZ, QF, DWS and YF conceived the idea and conceptualised the study. XLL, LNY, LYS and CYW, collected the data. FZ, XLL and HMY analysed the data. FZ, QF, DWS and YF drafted the manuscript, then DWS and QF, reviewed the manuscript. All authors read and approved the final draft.

\section{Funding}

This study was supported by a grant from Projects: Health science and Technology plan projects of Yunnan Province (Nos. 2017NS214, 2017NS215, 2017NS216, 2018NS0235, 2018NS0236); Provincial innovation team project of human assisted reproductive technology research: 2017 HC009. Martin expert workstation (2018IC106). Famous doctors of 'ten thousand people plan' in Yunnan Province (No. YNWR-MY-2018-014); Yunnan reproductive genetic disease clinical medical center (ZX2019-01-01); National Natural Science Foundation of China (No. 31700798). The funding body had no role in the design of the study and collection, analysis, and interpretation of data and in writing the manuscript.

\section{Availability of data and materials}

The datasets used and/or analysed during the current study available from the corresponding author on reasonable request.

\section{Ethics approval and consent to participate}

This study was conducted with approval from the Ethics Committee of the First People's Hospital of Yunnan Province and Dehong Dai and Jingpo Autonomous Prefecture Hospital. This study was conducted in accordance with the declaration of Helsinki. Ethics committee ruled that informed consent was not required in this study. Don't required any administrative permissions to access and use the medical records described in my study.

\section{Consent for publication}

Not applicable.

\section{Competing interests}

The authors declare that they have no competing interests.

\footnotetext{
Author details

${ }^{1}$ Department of Obstetrics and Gynecology, The First People's Hospital of Yunnan Province, No. 157 of Jinbi Road, Xishan District, Kunming 650032, China. ${ }^{2}$ Medical School of Kunming University of Science and Technology, Kunming 650500, China. ${ }^{3}$ Kunming Dianchi Lake Evironmental Protection Collaborative Research Center, Kunming University, Kunming 650214, China. ${ }^{4}$ Department of Obstetrics and Gynecology, Peking Union Medical College Hospital (Dongdan Campus), No.1 Shuaifuyuan Wangfujing, Dongcheng District, Beijing 100730, China. ${ }^{5}$ Department of Gynecology, Dehong Hospital of Traditional Chinese Medicine, Mangshi 678400, China. ${ }^{6}$ Department of Gynecology, The Second People's Hospital of Baoshan City, Baoshan 678000,
} 
China. ${ }^{7}$ Department of Obstetrics and Gynecology, Cangyuan Wa Autonomous County People's Hospital, Cangyuan 677400, China.

Received: 3 June 2020 Accepted: 9 February 2021

Published online: 01 March 2021

\section{References}

1. Tamrakar SR, Bastakoti R. Determinants of Infertility in Couples. J Nepal Health Res Counc. 2019:17:85-9.

2. Cunningham J. Infertility: a primer for primary care providers. JAAPA. 2017:30(9):19-25.

3. Jeje EA, Alabi TO, Ojewola RW, Ogunjimi MA, Osunkoya SA. Male infertility: an audit of 70 cases in a single centre. Afr J Urol. 2016;22:223-6.

4. Zargar AH, Wani Al, Masoodi SR, Laway BA, Salahuddin M. Epidemiologic and etiologic aspects of Primary infertility in the Kashmir region of India. Fertil Steril. 1997;68:637-43.

5. Yilmaz T, Yazici S, Benli T. Factors associated with infertility distress of infertile women: a cross-sectional study. J Psychosom Obstet Gynaecol. 2020;41(4):275-81.

6. Malekshah AK, Moghaddam AE, Moslemizadeh N, Peivandi S, Barzegarnejad A, Musanejad N, et al. Infertility in Mazandaran Province-north of Iran: an etiological study. Iran J Reprod Med. 2011;9:21-4.

7. Spain J, Rheinboldt M. MDCT of pelvic inflammatory disease: a review of the pathophysiology, gamut of imaging findings, and treatment. Emerg Radiol. 2017;24:87-93.

8. Brunham RC, Gottlieb SL, Paavonen J. Pelvic inflammatory disease. N Engl J Med. 2015;372:2039-48.

9. Loeper N, Graspeuntner S, Rupp J. Microbiota changes impact on sexually transmitted infections and the development of pelvic inflammatory disease. Microbes Infect. 2018;20:505-11.

10. Moran LJ, Misso ML, Wild RA, Norman RJ. Impaired glucose tolerance, type 2 diabetes and metabolic syndrome in Polycystic ovary syndrome: a systematic review and meta analysis. Hum Reprod Update. 2010;16:347-63.
11. Mitchell C, Prabhu M. Pelvic inflammatory disease: current concepts in pathogenesis, diagnosis and treatment. Infect Dis Clin North Am. 2013;27:793-809.

12. Zhang J, AiXingZi AL, Ding Y. Research progress of endometriosis infertility. Chin J Clin Obstet Gynecol. 2010;11:315-7.

13. Jiao XZ. Diagnosis and treatment of pelvic inflammatory diseases in primary hospitals. Chin Remed Clin. 2013;10:907-8.

14. Fitzgerald S, DiVasta A, Gooding H. An update on PCOS in adolescents. Curr Opin Pediatr. 2018:30:459-65.

15. Glueck CJ, Goldenberg N. Characteristics of obesity in polycystic ovary syndrome: etiology, treatment, and genetics. Metabolism. 2019;92:108-20.

16. Liu N, Liu GP. Laparoscopic salpingoplasty for hydrosalpinx infertility. Chin J Clin Obstet Gynecol. 2013;29:500-3.

17. Guan J, Watrelot A. Fallopian tube subtle pathology. Best Pract Res Clin Obstet Gynaecol. 2019:59:25-40.

18. Robinson J, Colimon LM, Isaacson KB. Postoperative adhesiolysis therapy for intrauterine adhesions (Asherman's syndrome). Fertil Steril. 2008;90:409-14.

19. McWilliams GD, Frattarelli JL. Changes in measured endometrial thickness predict in vitro fertilization success. Fertil Steril. 2007;88:74-81.

20. Barker MA, Boehnlein LM, Kovacs P, Lindheim SR. Follicular and luteal phase endometrial thickness and echogenic pattern and pregnancy outcome in oocyte donation cycles. J Assist ReProd Genet. 2009;26:243-9.

21. Zhang XD, Kennedy E, Temmerman M, Li Y, Zhang WH, Luchters S. High rates of abortion and low levels of contraceptive use among adolescent female sex workers in Kunming, China: a cross-sectional analysis. Eur J Contracept Reprod Health Care. 2014;19:368-78.

\section{Publisher's Note}

Springer Nature remains neutral with regard to jurisdictional claims in published maps and institutional affiliations.
Ready to submit your research? Choose BMC and benefit from:

- fast, convenient online submission

- thorough peer review by experienced researchers in your field

- rapid publication on acceptance

- support for research data, including large and complex data types

- gold Open Access which fosters wider collaboration and increased citations

- maximum visibility for your research: over $100 \mathrm{M}$ website views per year

At $\mathrm{BMC}$, research is always in progress.

Learn more biomedcentral.com/submissions 